\title{
Stabilization and Clustering Nodal Mechanisms in the Wireless Sensing Network
}

\author{
Deepti Narang \\ M.Tech. Student, Department of ECE, \\ Maharishi Markandeshwar University, Mullana, \\ Haryana, India.
}

\author{
Vijay Kumar \\ Asst. Professor, \\ Department of ECE, Maharishi Markandeshwar \\ University, Mullana, Haryana, India
}

\begin{abstract}
This paper presents the clustering mechanism implemented across the sensing nodes of Wireless Networks. The paper discusses the nodal architecture of sensing network and the issues which raise the instabilities in Network processes during its implementation in practical environment. Also the standard routing protocols are also described and evaluated for the node lifetime span. Efficient clustering mechanisms are essential for ensuring network's maximum life and uninterrupted service delivery. The routing protocols implemented are: Low Energy Adaptive Clustering Hierarchy, Stable Election Protocol, and Deterministic Energy-efficient Clustering. These models are simulated with standard parameters that provide the practical exposure of Wireless network to the system. The results are stated for each model respectively. The key solutions promised in each protocol are individually considered besides exploring the literature, for making network more stable and energy efficient.
\end{abstract}

\section{Keywords}

Sensor Networks; Network stability; Clustering mechanism; Sensor Nodes; Wireless Sensing Network.

\section{INTRODUCTION}

The twenty-first century technology has evolved the communication networks to much independent level where communication systems are equipped with self maintenance subsystems to analyse their operations as well as operational environment on their own. Modern communication networks have micro sensing terminals called sensor nodes which are deployed across the networks' physical structure for monitoring geographical and environmental phenomena like humidity, temperature, network energy, structural vibrations etc. in local as well as wide area network [1]. The sensing networks measure ambient environmental parameters from the network's surroundings and transform them into an electrical signal. Wireless Sensor Networks (WSN) can outlined as a network consists of VLSI and Nano scale based complex devices referred as sensor nodes that may sense the environment or surroundings and gather the knowledge from the observance field and communicate through wireless links; the information collected is forwarded, via multiple hops relaying to a sink (also referred to as controller or monitor) that may use it locally, or is connected to alternative networks [2]. The sensor nodes are typically scattered in a sensor field as shown in Fig. 1. Every of those scattered sensor nodes has the capabilities to gather information and route information back to the sink and also the end users. Information is routed back to the end/finish user by multichip infrastructure design through the sink as shown in Fig. 1. The sink could communicate with the task manager node via web/internet or Satellite [3]. Designing protocols and applications for such networks needs to be energy aware so as to prolong the lifetime of the network, as a result of the substitution of the embedded batteries may be a terribly troublesome method once these nodes are put in or installed. Sensors usually link the physical world with the digital world by capturing and revealing real-world development and changing these into a kind that may be processed, stored, etc. Sensors offer remarkable or incredible benefits when integrated into varied devices, machines, and environments [4]. They will assist to avoid catastrophic infrastructure failures, conserve precious natural resources, boost productivity, improve security, and upgrade new applications such as context-aware systems and smart home technologies. The miniaturization of computing and sensing technologies allows the development of small, low-power, and cheap actuators, sensors, and controllers.

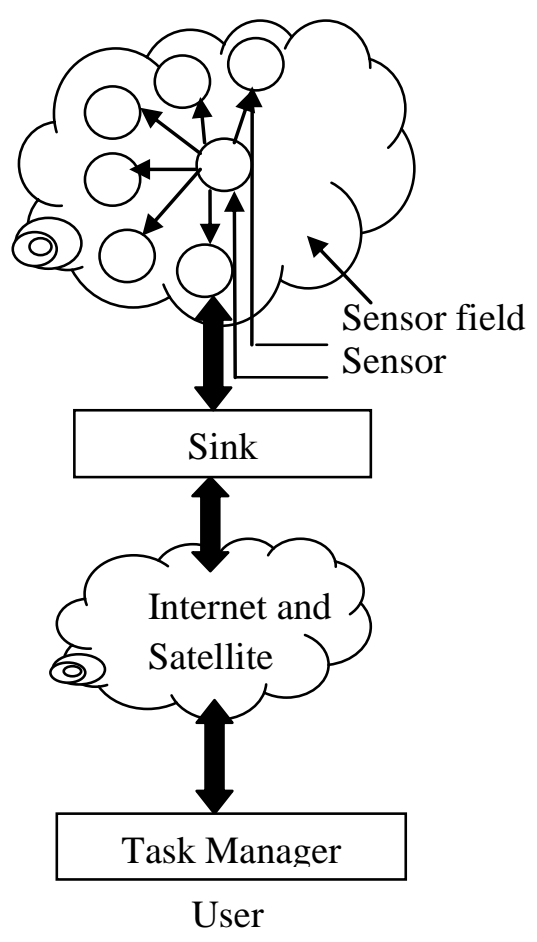

Figure 1. Sensor nodes spread in a sensor field 


\section{FUNDAMENTALS OF SENSOR NETWORKS}

Sensing is a very important technique that is used to gather information about a physical object or process including change in the state such as drop in temperature or humidity variations. From a technical perspective, a sensor may be a device that translates parameters or events within the physical world into signals that may be measured and analyzed [4]. At structural level a sensor node has three basic components: the sensing unit that collects data from the surrounding environment, the computing unit for processing the formerly collected data and later storing that data, and the wireless communication unit that deals with transmission of data. Also some power source is also required by the sensing node to perform its operations. This power source used in sensor networks is a battery with limited energy that cannot be recharged or replaced. Although network can be deployed in any harsh environment conditions across the globe and the sensor network must have enough lifetime to serve the cause of deployment and met all needs of the respective application.

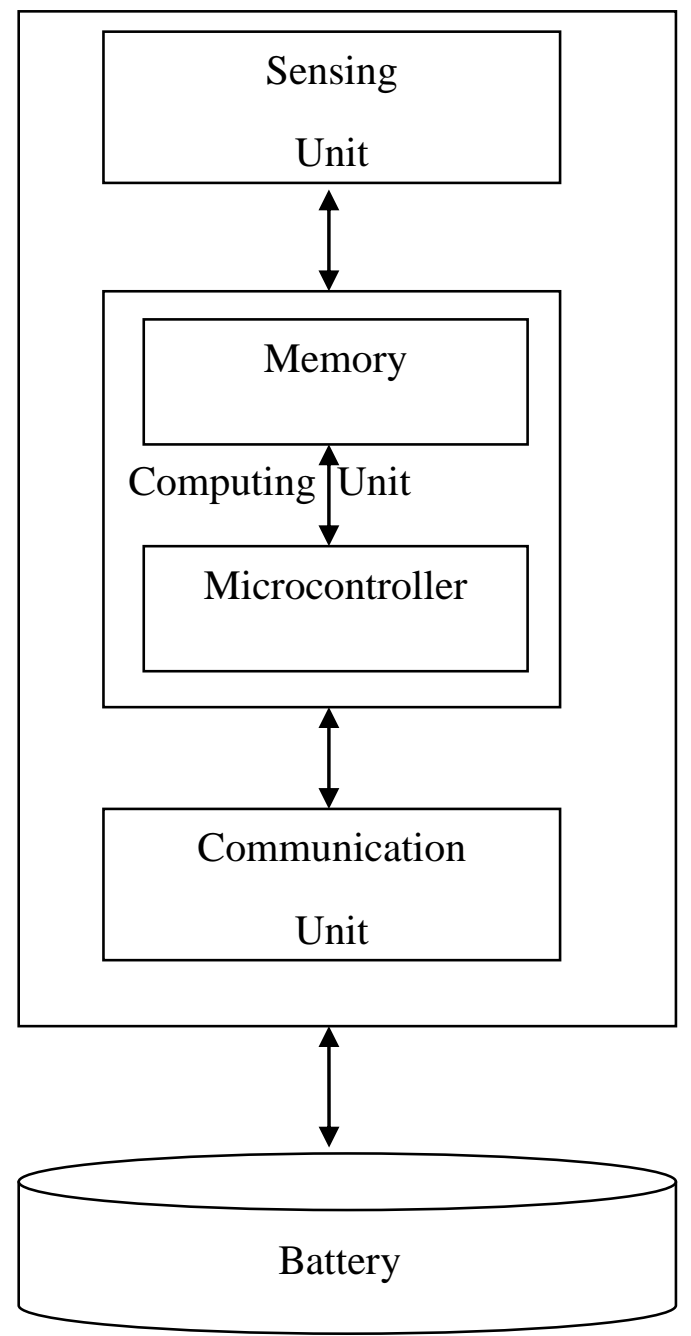

Figure 2. Block Diagram of Sensor Node
When multiple sensors are grouped to monitor giant physical environments like as in wireless sensor networks, the clustered sensors function as a individual computing and processing system capable of diagnosing its parameters besides the main networks' statistics. Networks implementing such clustered sensor nodes are usually deployed to monitor giant geographic areas, environmental pollution control measures, monitoring sensitive concrete structure, healthcare applications etc. The idea of deployment of Sensor Networks in agriculture and food industry has attracted considerable research efforts in the present scenario of crop destruction in fields and storage places due to lack of knowledge of accurate environment parameters. WSNs are highly precise for distributed data collecting in tough environments such as greenhouses, warehouses, cropland and also can be used in detecting the environmental variations so that the farmers and warehouse employees can be timely alerted [5].

\section{INSIGHT OF SENSING TECHNOLOGY}

The basic functionality of a network's Sensor Nodes is collecting the information from the environment and sending it to the selected cluster head which further transmit information to the base station. The intera-nodal communication in Sensor Networks follows specific Routing algorithms analogous to the computer communication networking. These routing protocols in Sensor Networks can be classified into two categories on the basis of network structure as: flat routing and clustering routing. The flat routing protocol implies that all nodes have same functionalities and perform same tasks and the data transmission is achieved in multiple hops while in the clustering or hierarchical protocol, nodes have different tasks and are divided into groups called clusters [8]. In each cluster there is a cluster head $(\mathrm{CH})$ which communicates with base station (BS), and other nodes called member nodes which collect and send data to the cluster head as shown in the diagram below Figure3. The clustering protocols exhibit certain network issues [6]. The principle followed in the cluster head selection in each of routing protocol is that it must be energy efficient and capable of stabilizing the network [9].

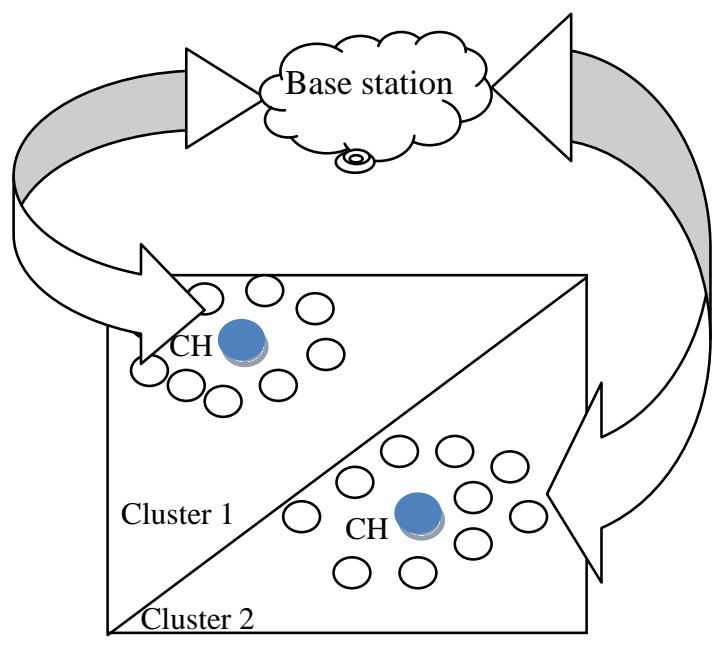

Figure3. Cluster Head Selection Mechanism 


\subsection{Cluster head selection :}

In this phase, several cluster heads are elected. Nodes become CLIENT nodes with a probability $T$ and then broadcast the COMPETE HEAD MSGs within radio range $R$ compete to advertise their wills. Each CLIENT node checks whether there is a CLIENT node with more residual energy within the radius $R$ compete. Once the CLIENT node finds a more powerful CLIENT node, it will give up the competition without receiving further COMPETE HEAD MSGs. Otherwise, it will be elected as HEAD in the end.

\subsection{Cluster building:}

In this phase, each HEAD node broadcasts the HEAD AD MSG across the network, while the CLIENT nodes receive all the HEAD AD MSGs and decide which cluster to join. Most of existed metric for CLIENT nodes to make decisions is the distance metric. For example if the CLIENT nodes choose the cluster head that require minimum communication according to the received signal strength [10][11]. However, pursuing effcient energy consumption of the CLIENT nodes only may lead HEAD nodes exhausted quickly during the data transmission phase. In the data transmission phase, the consumed energy of cluster head i, E(CHi) is as follows, assuming : $d(C H i ; B S)>d c r o s s o v e r$.

$$
E(C H i)=\text { milEelec }+(m i+1) l E D A+l\left(\text { Eelec }+{ }^{2} m p d 4\right)
$$

Observing energy consumption of $E(\mathrm{CHi})$ is composed of three parts: data receiving, data aggregation and data transmission. In the field, several cluster heads may be near the BS, while some are far away. The energy expended during data transmission for far away cluster heads is significant, especially in large scale networks. Since $d(\mathrm{CH} ; ; \mathrm{BS})$ has been fixed after cluster head election, we should justify the cluster size for each cluster head to balance their load across the network. The larger $d(\mathrm{CHi} ; \mathrm{BS})$ is, the smaller member size $m i$ the cluster head $C h i$ should accommodate. Energy consumption of the CLIENT node $P j$ during transmitting the data to $\mathrm{CHi}$ obey the above expression 1 . Let $E(P j)$ be the energy consumed by $P j$. If $P j$ always chooses the cluster head CHbest with min $f E(P j) g$, CHbest may be exhausted due to long distance data transmission to the BS and immoderate cluster size, although the energy of $P j$ is saved. Thus, CLIENT node $P j$ in EECS chooses the cluster head by considering not only saving its own energy but also balancing the workload of cluster heads,i.e. two distance factors: $d(P j$ $; \mathrm{CHi})$ and $d(\mathrm{CHi} ; \mathrm{BS})$. We introduce a weighted function $\operatorname{cost}(j ; i)$ for the CLIENT node $P j$ to make a decision, which can be stated as:

$$
\operatorname{cost}(j ; i)=w f f(d(P j ; C H i))+(1 ; w) £ g(d(C H i ; B S)) ;
$$

and $\mathrm{Pj}$ chooses $\mathrm{CHi}$ with min $f$ costg to join. In above expression, $f$ and $g$ are two normalized functions for the distance $d(\mathrm{Pj} ; \mathrm{CHi})$ and $d(\mathrm{CHi} ; \mathrm{BS})$ respectively:

$$
\begin{gathered}
f=d(P j ; \text { Chi }) / d \text { fmax } \\
g=d(\text { Chi } ; B S)-\text { dgmin/dgmax }- \text { dgmin }
\end{gathered}
$$

where $d f \max =E X(\max f d(P j ; C H i) g), d g \max =\max$ $f d(C H i ; B S) g$ and $d g \min =\min f d(C H i ; B S) g . f$ subfunction in cost guarantees that members choose the closest cluster head in order to minimize energy consumption of the cluster members, While $g$ subfunction makes the nodes join the cluster head with small $d(\mathrm{CHi} ; \mathrm{BS})$ to alleviate the workload of the cluster heads farther from the BS.

\subsection{Synchronization and design issues:}

Synchronization between each phase should be guaranteed that each node has enough time to complete the procedure; while within each phase, synchronization among the nodes is not necessary and idle nodes will turn to sleep till the phase ends. In EECS, it is achieved by having the BS periodically broadcast synchronization signals to all nodes.

\section{NETWORK ROUTING PROTOCOLS}

Some widely accepted routing protocols that have become common standards of Clustering mechanism in sensing network are described here with their merits and demerits in practical network environment.

\subsection{LEACH protocol:}

Low Energy Adaptive Clustering Hierarchy (LEACH) is one of the mostly used hierarchical cluster-based routing protocols for wireless sensor network [12]. In LEACH as mentioned above the network is divided into number of clusters which uses TDMA (Time Division Multiple Access) schedule for member nodes. This protocol consists of number of rounds $r$ and each round has two phases: set up phase and steady-state phase. In set up phase Cluster Head $(\mathrm{CH})$ selection is based on two factors. First, the percentage $\mathrm{P}$ of nodes in network and secondly history of nodes that has served as $\mathrm{CH}$. A threshold value $T(n)$ is set and decision is made by each node $n$ based on the random number between 0 and 1 . The value of $T(n)$ is given by expression:

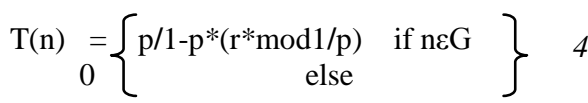

The generated random number is if less then threshold value then node becomes cluster head for that round. Thereafter, each node has probability $1 / \mathrm{p}$ of becoming $\mathrm{CH}$ in each round. In steady-state phase, nodes send their collected data by using their allocated TDMA slot to $\mathrm{CH}$. The $\mathrm{CH}$ aggregates the data when received and send it to the Base Station (BS). There are some problems associated with LEACH Protocol: The cluster head is selected randomly so each node in cluster has same probability to become cluster head. After numerous rounds, node with high remaining energy and node with low remaining energy have same probability of becoming cluster head. If the node with low remaining energy is chosen as cluster head, it will run out of energy and die quickly which affects network lifetime. The division of clusters is also done in random fashion which results in uneven distribution of clusters. The divided clusters have non uniformities in terms of nodes or location of cluster heads at centre or at edge of cluster. These non uniformities affect network's performance. Recent researches such as Two Phase Clustering Method for cluster head selection has also been done in order to improve the LEACH protocol [12]. In this research, a two stage clustering protocol based on Self Organizing Map neutral network and Modified Fuzzy Probabilistic Clustering Algorithm for balancing the energy consumption. The clustering is based on two important conditions, coordinates of sensor nodes and energy level. Thus, this two stage clustering method can prevent the premature death of nodes.

\subsection{PEGSIS Protocol:}

Power Efficient Gathering in Sensor Information System (PEGASIS) is an improved version of LEACH protocol [12]. This protocol is guaranteed by two characteristics only one node communicates with base station at a time and rest of the nodes communicates only with their neighbor [13]. Each node 
communicates with nearest neighbor by adjusting its power signal strength. In this way, each node measure distance to the neighborhood nodes in order to find the nearest node. In this process a chain is formed (Figure 2.1) and a leader is elected from chain on the basis of residual energy in every round. The head collects data from neighbours and transmits the collected data to base station. Thus average energy consume by each node per round is decreased. This approach also reduces bandwidth requirement by lowering the overhead required.

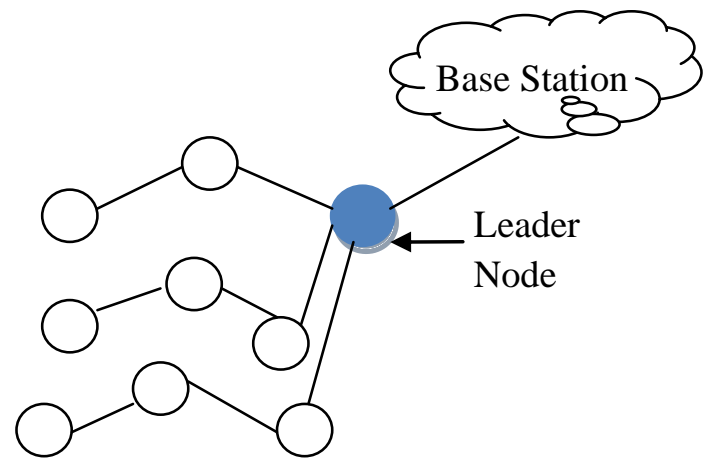

Figure 4. PEGASIS protocol

The PEGASIS also has limitations for example: it is complicated task for all nodes to maintain a complete database about the location of all other nodes within the network and also the communication in PEGASIS suffers from excessive delays caused by single chain for distant nodes and a high probability for any node to become a bottleneck. Recent study by author in proposed the Hop PEGASIS approach which is more efficient than the LEACH and PEGASIS[13][14]. If there is a direct transmission between $\mathrm{CHs}$ and base station, the cluster head that is situated not much far away from the base station uses strong signals while transmission to the base station thus leads to less energy consumption, so in this protocol the lifetime of network gets improved. The experimental results shows that, in hop PEGASIS the nodes survived around 3000 rounds better than LEACH (1100 rounds) as well as PEGASIS (2000 rounds).

\subsection{HEED Protocol:}

Hybrid Energy Efficient Distributed Clustering (HEED) protocol is an extension of LEACH protocol, in this protocol the residual energy is used as primary parameter while other topology features like node degree, distances to neighbors are used as secondary parameter to attain power balancing in network. The clustering process is split into number of iterations, in every iteration nodes that are not covered by any cluster head and thus doubles their probability of becoming a cluster head. As these energy-efficient clustering protocols further enables each node to probabilistically and independently decide its role in the clustered network. Moreover they cannot guarantee optimal elected set of cluster heads [15]. This protocols introduced uniform Cluster head distribution across the network by enabling low power levels of cluster put in spatial reuse while high power levels of clusters are used for inter-cluster communication. The long range communication from $\mathrm{CHs}$ to sink is possible as $\mathrm{CHs}$ and BS communication has become energy efficient.

The main limitation of HEED is that it imposes significant overhead in network causing remarkable energy dissipation which results in reduced network lifetime. The $\mathrm{CHs}$ near the sink might die earlier because of high workload on them and also the uncertain $\mathrm{CHs}$ that do not become final $\mathrm{CHs}$ leave some uncovered nodes, these nodes are forced to become $\mathrm{CH}$ and these forced $\mathrm{CH}$ may not have any member associated with them. As result, unexpected number of $\mathrm{CHs}$ are generated which unbalances energy consumption of network. The heterogeneous HEED (H-HEED) has been proposed, this protocol basically used in heterogeneous WSNs. The recent developments in H-HEED also serve purpose to prolong network lifetime and energy efficiency [15]. The simulation results show that the throughput and packet delivery ratio are improved by using H-HEED and the delay and energy consumption is decreased.

\subsection{SEP Protocol:}

Stable Election Protocol (SEP) is also a further modification to LEACH protocol. In this protocol two types of nodes and two level hierarchies are considered. It is based on weighted electron probabilities of each node to become cluster head according to remaining energy in each node. SEP is a heterogeneous-aware protocol and it prolongs the time interval before the death of first node which is referred as stability period [16]. In order to prolong stable region, SEP maintain a well balance of energy consumption. The advanced nodes have to become cluster heads earlier than normal nodes. The cluster head election is arbitrarily selected and distributed based on the fraction of energy of every node assuring a uniform use of the nodes energy.

The main limitation of SEP is that election of cluster heads among the two type of nodes is not dynamic, which results that the nodes that are far away from the powerful nodes will die first. The improvement to SEP protocol is also introduced known as fuzzy logic approach to improving stable election protocol [16]. The SEP- FL (SEP by Fuzzy Logic) is based on two criteria: the distance from the base station and the residual energy level of each node type. The SEP-FL increases the stability period and decreases the instability of the sensor network as compared with LEACH and SEP. This protocol provides longer interval of stability for large values of additional energy brought by advanced nodes.

\subsection{DEC Protocol:}

Deterministic Energy-efficient Clustering (DEC) protocol is a purely deterministic model that utilizes clustering to organize WSN. This protocol is dynamic, distributed, self-organizing and more energy efficient than existing protocols. The approach of this protocol is simple that is to minimize computational overhead-cost to self-organize the network. This protocol has better performance with respect to energy consumption in both heterogeneous and homogeneous network. This protocol uses residual energy of each node in the cluster for election process of $\mathrm{CH}$. The uncertainties in the cluster-head elections have been minimized in DEC. The setup phase used in LEACH is modified, but the steady-state phase is kept same as that of in LEACH protocol. Since node's energy can be determined a priori, the $\mathrm{CH}$ election process is reorganized by using the RE of each node [17]. In $\mathrm{DEC}$, the BS elects no. of cluster-heads at round $\mathrm{m}$ for the network. The BS can only take part in the election of $\mathrm{CHs}$ if and only if $m=1$. The elected $\mathrm{CHs}$ advertise their role using CSMA MAC just as in LEACH. However, in DEC unlike in $\mathrm{LEACH}$, the join-request message will contain CM-ID (Cluster member ID), CH-ID (Cluster Head ID) and CM-RE (cluster member-residual energy) and the header that indicates it as a request. This way the RE information of CMs is known to their respective $\mathrm{CHs}$ thus localized and it can be utilized for $\mathrm{CH}$ rotation in the subsequent rounds. Thus the establishment of a Heterogeneous wireless sensor network will be done by 
using DEC routing protocol to increase the network lifetime and to reduce the energy consumption.

\section{SIMULATION}

For simulation model, network is designed to work in both heterogeneous and homogeneous environment, thus there are three levels of nodes defined in on basis of their energy level, these are as: Normal nodes $(\mathrm{O})$, Intermediate nodes $(\boldsymbol{\omega})$ and Advanced nodes $(\boldsymbol{\gamma})$. In the implementation procedure, a multilevel clustering model is employed in which the nodes with different energy levels are randomly deployed within a space region called field. The values of initial parameters are shown in Table 2. In first step the sink or base station is plotted with coordinates at the centre of field, as shown in Figure 5. It is to be noted that the distance on $\mathrm{X}$ and $\mathrm{Y}$ coordinates in Figure 5 is in meters. The size of field is $100 \mathrm{x}$ $100 \mathrm{~m}$, the Figure 5 illustrates the location of the sink.

Table 1: Parameters In Network Simulation.

\begin{tabular}{|c|l|l|}
\hline Parameter & \multicolumn{1}{|c|}{ Description } & \multicolumn{1}{|c|}{ Value } \\
\hline $\mathrm{xm}$ x ym & Dimensions of Field & $100 \mathrm{~m} \mathrm{x} \mathrm{100m}$ \\
\hline $\mathrm{n}$ & No of Nodes & 100 \\
\hline $\mathrm{rmax}$ & Max no of Rounds & 4000 \\
\hline $\mathrm{p}$ & $\begin{array}{l}\text { Probability of node to } \\
\text { become CH }\end{array}$ & 0.1 \\
\hline Eo & $\begin{array}{l}\text { Initial energy of each } \\
\text { node }\end{array}$ & $0.5 \mathrm{~J}$ \\
\hline ETX & $\begin{array}{l}\text { Transmission energy } \\
\text { of node }\end{array}$ & $\begin{array}{l}50 * 0.00000000 \\
1 \mathrm{~J}\end{array}$ \\
\hline ERX & $\begin{array}{l}\text { Receiving energy of } \\
\text { node }\end{array}$ & $\begin{array}{l}50 * 0.00000000 \\
1 \mathrm{~J}\end{array}$ \\
\hline EDA & $\begin{array}{l}\text { Data aggregation } \\
\text { energy }\end{array}$ & $\begin{array}{l}5 * 0.000000001 \\
\mathrm{~J}\end{array}$ \\
\hline Efs & $\begin{array}{l}\text { Energy dissipation } \\
\text { for free space }\end{array}$ & $\begin{array}{l}10 * 0.00000000 \\
0001 \mathrm{~J}\end{array}$ \\
\hline Emp & $\begin{array}{l}\text { Energy dissipation } \\
\text { for multi-path delay }\end{array}$ & $\begin{array}{l}0.0013 * 0.00000 \\
0000001 \mathrm{~J}\end{array}$ \\
\hline Packet & Packet size & 4000 \\
\hline & \multicolumn{2}{|l}{} \\
\hline
\end{tabular}

In next step the node deployment is done. To simulate heterogeneous environment, there are three level clustering model of standard DEC. The parameters associated with multilevel clustering model are given in TABLE II. In next step clustering is done, where a cluster is a group of nodes in which one node act as leader and other nodes act as member nodes. The clustering technique is shown in Figure 9. The nodes which act as cluster heads are represented by pointer symbol on them. At the end of each round the cluster head becomes changed. The clusters are formed on basis of minimum routing distance procedure. The distance between nodes is calculated by distance formula:

$$
D=\sqrt{ }\left((x 1-x 2)^{2}+(y 1-y 2)^{2}\right)
$$

Where (x2, y2) and (x1, y1) are coordinates of two nodes plotted at some distance each other. (one is member node and other is cluster head)
Table 2: Parameters Associated with Clustering.

\begin{tabular}{|l|l|}
\hline Parameter & Value \\
\hline Percentage of normal nodes & $50 \%$ of Total nodes \\
\hline $\begin{array}{l}\text { Percentage of intermediate } \\
\text { nodes }\end{array}$ & $30 \%$ of Total nodes \\
\hline Percentage of advance nodes & $20 \%$ of Total nodes \\
\hline
\end{tabular}

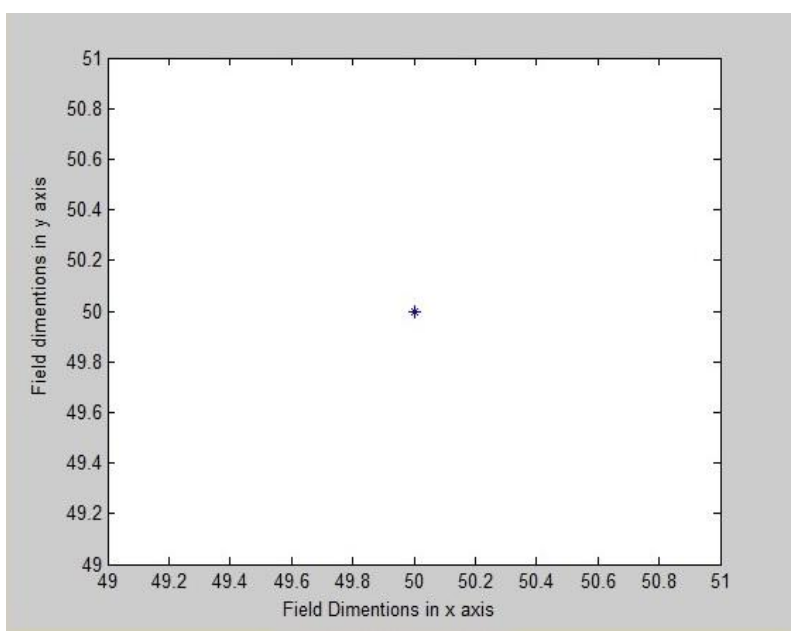

Figure 5. Plot of sink/base station at centre of the field.

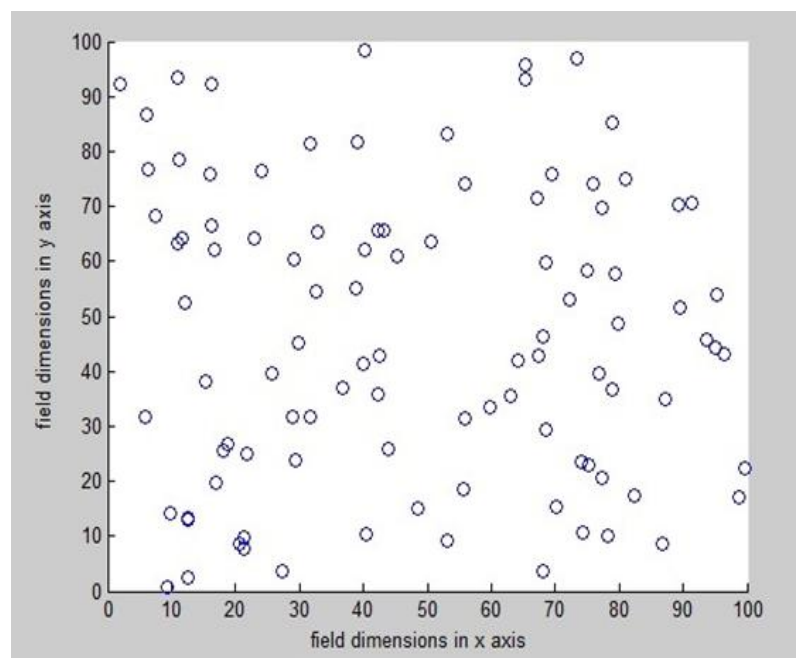

Figure 6. Energy $=0.5 \mathrm{~J}$ per node and Node percentage $=$ $50 \%$.

In Figure 7, the nodes with remaining energy less than half are shown. After some subsequent rounds the nodes energy starts depleting and when a node's remaining energy is less than half then these nodes are represented by pink color. The dead nodes are shown by red color as shown in Figure 10. 


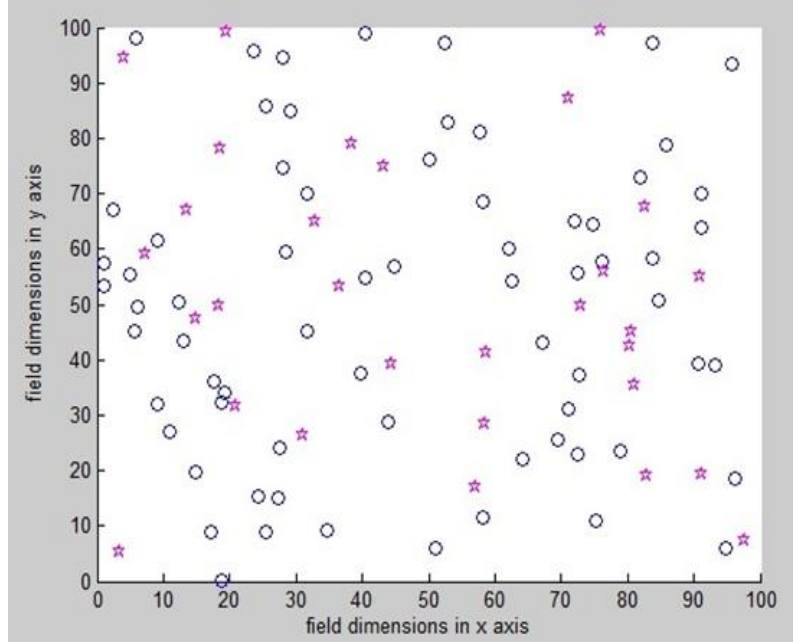

Figure 7.Energy $=1.25 \mathrm{~J}$ per node and Node percentage $=$ $30 \%$.

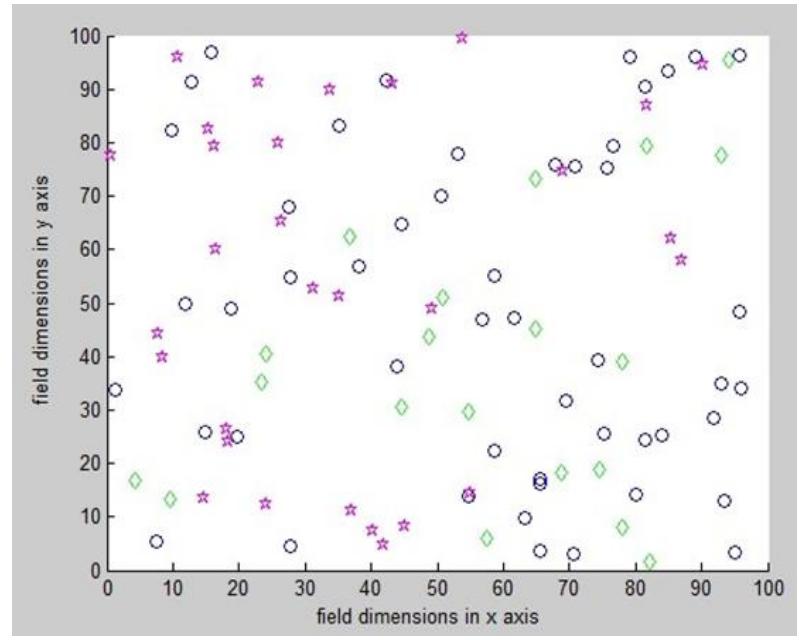

Figure 8.Energy $=2.0 \mathrm{~J}$ per node and percentage nodes $=$ $20 \%$.

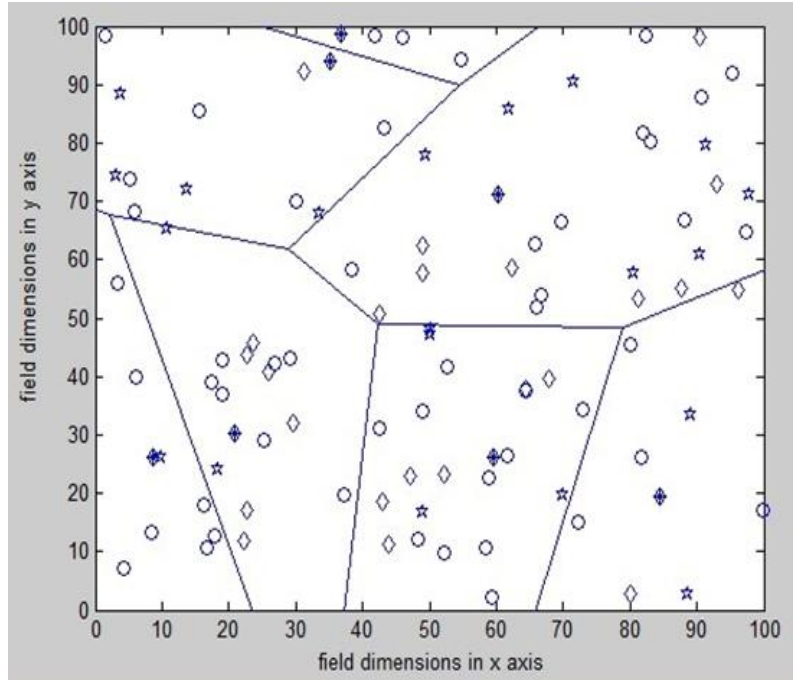

Figure 9.Cluster Heads represented by (*) during Clustering.

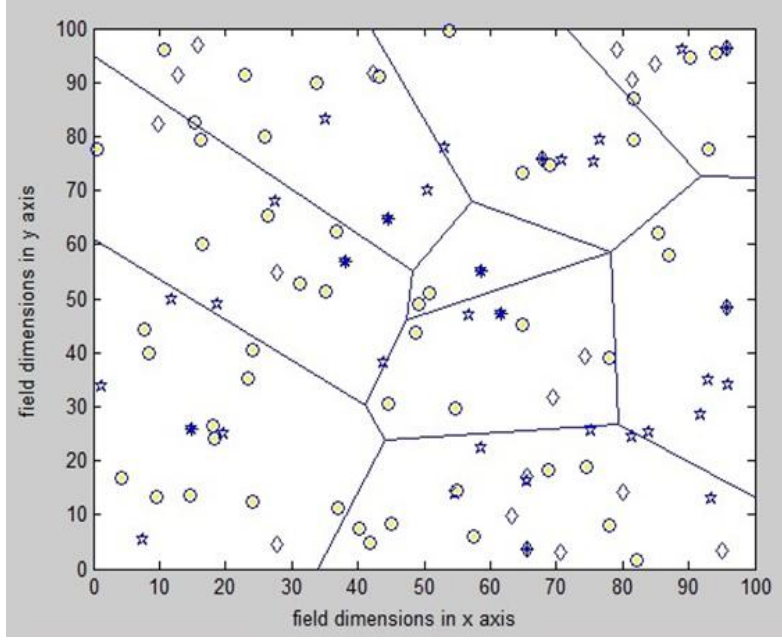

Figure 10.Energy level reaches to $0 \%$ and nodes becoming dead

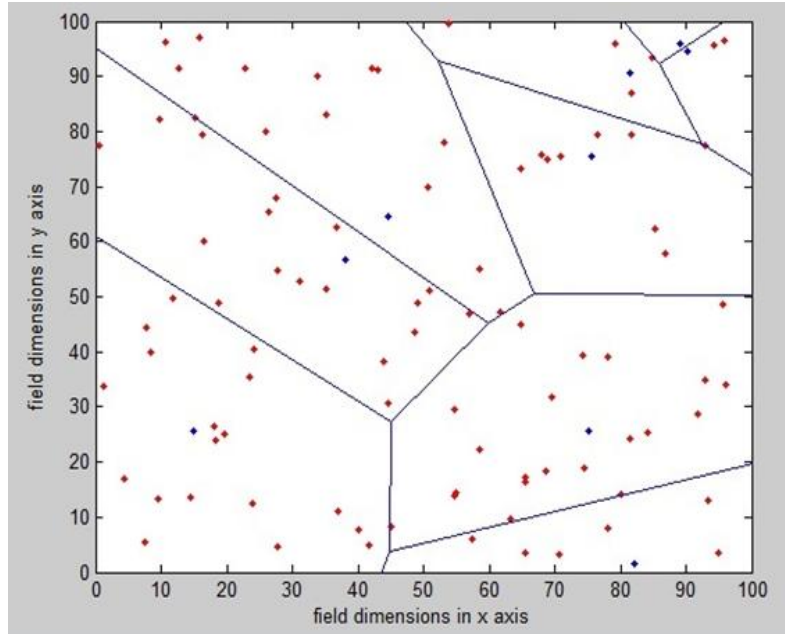

Figure 11. Network instability due to death of node.

\section{RESULTS}

The Figure 12 shows alive nodes versus number of rounds comparative plot for DEC, ESEP and LEACH protocols. The Figure 13 shows dead nodes vs. number of rounds plot. This plot is for verification of alive nodes vs. number of round plot.

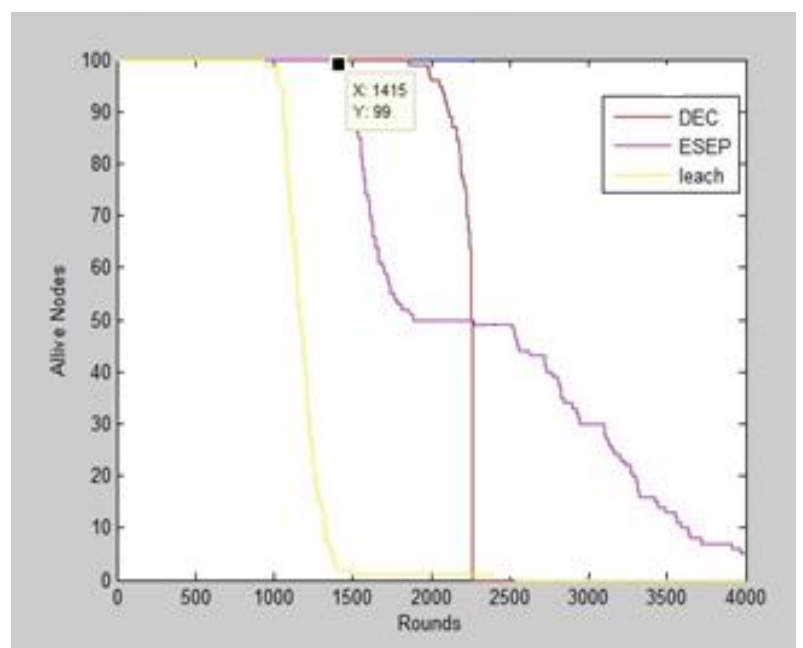

Figure 12. Alive nodes v/s rounds plot for standard routing protocols. 


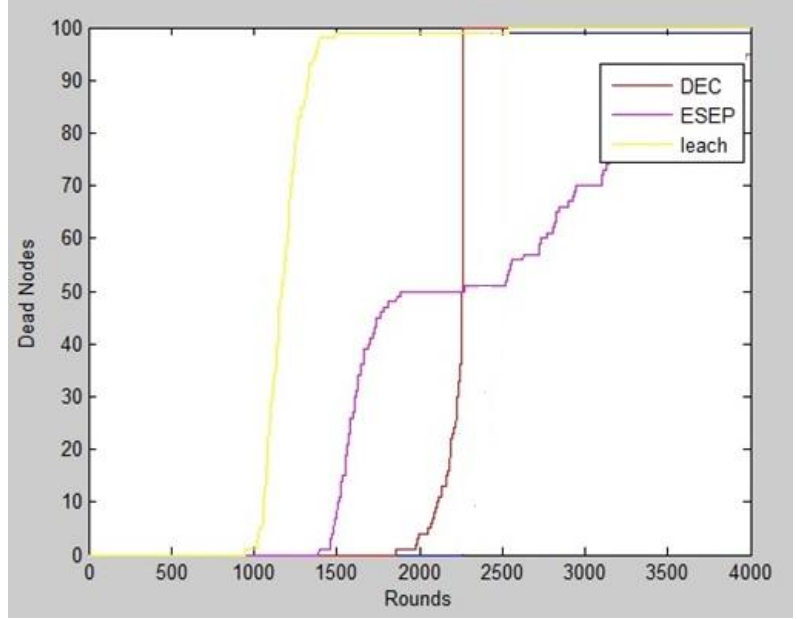

Figure 13. Dead nodes v/s rounds plot for standard routing protocols

\section{CONCLUSION}

The Sensor Networks technology is still in its initial phase evolution and holds the scope for a ton of research. The present day Sensing technology is application specific designs so the protocols designed should be such that it suits sensor network serving respective application. One of the main challenges in WSNs is energy efficiency which is solved by a cluster head selection mechanism used by a protocol. The clustering protocol used should be energy efficient and stable so that network performance would be increased. In this paper, we introduced some of the design issues, limitations and recent research works of clustering protocols for WSNs. As by studying various clustering protocols, it is revealed that a protocol which will have good performance under all scenarios and for all application is not possible because all the protocols have some limitations associated with them. However, DEC protocol has better results as compared to other protocols as it selects fixed number of cluster heads per round and thus guaranteeing better stability of Sensor Network.

\section{REFERENCES}

[1] Giuseppe Anastasi, Marco Conti, Mario Di Francesco, Andrea Passarella, "Energy conservation in wireless sensor networks: A survey", Elsevier, Volume 7, Issue 3, Pages 537-568, May 2009.

[2] Sasikumar M, Dr. R. Anitha, "Performance Evaluation of Heterogeneous-HEED Protocol for Wireless Sensor Networks", International Journal of Advanced Research in Computer and Communication Engineering, Vol. 3, Issue 2, February 2014.

[3] Md Arif Ali, Abha Kiran Rajpoot, "Development of energy efficient routing protocol using Hop PEGASIS in Wireless Sensor Networks", International Journal of Computer Science \& Engineering Technology (IJCSET), Vol. 5, No. 02, Feb 2014.

[4] Dr. Neeraj Bhargava, Dr. Ritu Bhargava, Shilpi Gupta, Kamal Kumar Jyotiyanain "Analysis of different congestion avoidance algorithm" International Journal of Computer Networks and Wireless Communication (IJCNWC), ISSN: 2550-3501. Vol.3,no1,Feb 2013.

[5] Luis Ruiz-Garcia, Loredana Lunadei, Pilar Barreiro and Jose Ignacio Robla, "A Review of Wireless Sensor
Technologies and Applications in Agriculture and Food Industry: State of the Art and Current Trends", Sensors, Volume 9, Issue 6, June 2009.

[6] Xuxun lio, "A Survey on Clustering Routing Protocols in Wireless Sensor Networks", MDPI, Sensors, volume 12, Issue 8, August 2012.

[7] Kiran Maraiya, Kamal Kant, Nitin Gupta, "Efficient Cluster Head Selection Scheme for Data Aggregation in Wireless Sensor Network",International Journal of Computer Applications (0975 - 8887) Volume 23- No.9, June 2011.

[8] Md. Habibe Azam1, Abdullah-Al-Nahid,Md. Abdul Alim, Md. Ziaul Amin, "A Survey and Comparison of Various Routing Protocols of Wireless Sensor Network (WSN) and a Proposed New TTDD Protocol Based on LEACH", International Journal of Computer and Network Security, Vol. 2, No. 8, August 2010.

[9] Rajashree.V.Biradar, V.C .Patil, Dr. S. R. Sawant, Dr. R. R. Mudholkar, "Classification and Comparison of Routing Protocols in Wireless Sensor Networks", ubicc Journal (Ubiquitous Computing and Communication Journal), Volume 4.

[10] W. Heinzelman, et. al., "An application-specific protocol architecture for wireless microsensor networks," IEEE Transactions on Wireless Communications, 1(4):660669, 2002.

[11] S. Bandyopadhyay, et. al., "An Energy-Effcient Hierarchical Clustering Algorithm for Wireless Sensor Networks," in IEEE INFOCOM'03.

[12] Rathi and Viswanathan, "Two Phase Clustering Method for Leach Protocol For Effective Cluster Head Selection", Journal of Computer Science, Science Publications 2014.

[13] S. Lindsey, C. Raghavendra, "PEGASIS: Power Efficient Gathering in Sensor Information Systems", IEEE Aerospace Conference Proceedings, Vol. 3, 9-16, pp.11251130, 2002.

[14] Md Arif Ali, Abha Kiran Rajpoot, "Development of energy efficient routing protocol using Hop PEGASIS in Wireless Sensor Networks", International Journal of Computer Science \& Engineering Technology (IJCSET), Vol. 5, No. 02, Feb 2014.

[15] O. Younis and S. Fahmy, "HEED: A Hybrid, EnergyEfficient, Distributed Clustering Approach for Ad Hoc Sensor Networks", IEEE Transactions on Mobile Computing, 3:366-379, 2004.

[16] G. Smaragdakis, I. Matta, A. Bestavros, "SEP: A Stable Election Protocol for clustered heterogeneous wireless sensor networks" In Second International Workshop on Sensor and Actor Network Protocols and Applications (SANPA), 2004.

[17] Femi A. Aderohunmu, Jeremiah D. Deng, Martin K. Purvis, "A deterministic Energy-efficient Clustering Protocol for Wireless sensor network", Information Science Department, University of Otago Dunedin, New Zealand, pages 341 - 346 Sensor Networks and Information Processing (ISSNIP), Dec. 2011. 